\title{
Increased serum levels of miR-214 in patients with PCa with bone metastasis may serve as a potential biomarker by targeting PTEN
}

\author{
YI FANG $^{1}$, JUN QIU ${ }^{2}$, ZONG-BIN JIANG ${ }^{3}$, SHENG-RONG XU $^{3}$, ZENG-HUA ZHOU $^{3}$ and RUI-LIN HE ${ }^{3}$ \\ ${ }^{1}$ Department of Anesthesiology, Changsha Central Hospital, Changsha, Hunan 410000; \\ ${ }^{2}$ Oncology Department Two, Mawangdui Hospital of Hunan People's Hospital, Changsha, Hunan 410016; \\ ${ }^{3}$ Department of Pain Medicine, Second Affiliated Hospital of Guangxi Medical University, \\ Nanning, Guangxi 530000, P.R. China
}

Received January 15, 2017; Accepted January 3, 2018

DOI: $10.3892 / 01.2018 .9522$

\begin{abstract}
MicroRNAs (miRNAs/miRs) are identified to serve key functions in the progression of various tumors. miR-214 is aberrantly expressed in various types of cancer. In the present study, the function of miR-214 and its feasibility as a potential non-invasive biomarker for patients with prostate cancer $(\mathrm{PCa})$ in a hyperplasia group and a control group were investigated. First, RNA was isolated from the serum of 75 patients with PCa with bone metastasis, 65 patients with PCa with no bone metastasis and 70 healthy controls. The level of miR-214 expression was significantly upregulated in the serum of the bone metastasis group compared with the healthy control and non-bone metastasis groups. Expression levels of alkaline phosphatase (ALP), bone sialoprotein (BSP), collagen type I pyridine crosslinking peptide (ICTP) were also evaluated. The results indicated that serum levels of BSP, ALP and ICTP were increased in the bone metastasis group compared with that in the non-bone metastasis group, hyperplasia group and the control group $(\mathrm{P}<0.05)$. The expression level of miR-214 is positively associated with poorly differentiated tumors in patients with $\mathrm{PCa}$ with a Gleason score $>7(\mathrm{P}<0.05)$. Western blot analysis demonstrated that phosphatase and tensin homolog (PTEN) was a target gene of miR-214. Additionally, silencing of PTEN significantly increased the invasive ability of PC3 cells even when miR-214 expression was inhibited. In summary, serum miR-214 expression may serve as a potential novel non-invasive biomarker for PCa screening through targeting PTEN.
\end{abstract}

Correspondence to: Dr Zong-Bin Jiang, Department of Pain Medicine, Second Affiliated Hospital of Guangxi Medical University, 6 Shuangyong Road, Nanning, Guangxi 530000, P.R. China

E-mail: zongbinjiang1011@163.com

Key words: microRNA-214, bone metastasis, prostate cancer, biomarker, phosphatase and tensin homolog

\section{Introduction}

In the last few years, prostate cancer (PCa) has become a common malignant tumor among men worldwide (1). It has been demonstrated that $\mathrm{PCa}$ is the second leading cause of cancer-associated mortality in western countries and it continues to increase (2). Bone metastasis in PCa may result in pathological fracture and bone pain, which poses a severe threat and may have an impact on quality of life and prognosis. Currently, radionuclide bone imaging is widely applied for the diagnosis and monitoring of $\mathrm{PCa}(3,4)$. However, radionuclide bone imaging exhibits low specificity and high cost, and there may be radioactive effects. Thus, it is imperative to explore alternative options for early diagnosis and treatment of bone metastasis in $\mathrm{PCa}$.

Serum markers demonstrated advantages in terms of reproducibility, non-invasiveness and relatively low cost. Previous studies have indicated that serum expression level of prostate-specific antigen (PSA) is an ideal marker for the prediction of the lesion range among patients with $\mathrm{PCa}$ (5). However, the level of PSA may be disturbed in other pathological conditions, including prostatitis and benign prostatic hyperplasia (BPH) (6), thus leading to over-diagnosis and overtreatment (7). Additionally, alkaline phosphatase (ALP) is also suggested to be an important predictor for bone metastasis (8). The expression level of serum bone sialoprotein (BSP) is an indicator of the bone resorption process and bone cell activity. Bone resorption marker collagen type I pyridine crosslinking peptide (ICTP) is considered to predict bone cell function and the bone absorption rate, which is also an important diagnostic marker in bone metastasis (3). However, these markers are increased in all patients with $\mathrm{PCa}$, indicating the necessity to identify specific markers for bone metastasis in patients with $\mathrm{PCa}$.

MicroRNAs (miRNAs/miRs) are small non-coding RNAs that are widely involved in post-transcriptional gene regulation through an incomplete base pairing mechanism $(9,10)$. Abnormal expression of miRNAs has been identified in various cellular processes, including cell differentiation, proliferation and apoptosis $(11,12)$. In the progression of bone metastasis in patients with PCa, abnormal expression of miRNAs is common $(13,14)$. For instance, epidermal growth factor receptor maintains the activation of oncogenic twist-related protein 1 mainly by 
suppressing miR-1, thereby accelerating bone metastasis (13). Furthermore, miR-34a may regulate Wnt/transcription factor 7 signaling and suppress bone metastasis in Ras-activated $\mathrm{PCa}$ cells (14).

The aim of the present study was to explore the function of miR-21. For instance, miR-214 was identified to be upregulated in gastric and pancreatic cancer $(15,16)$. Expression of miR-214 was decreased in renal carcinoma and glioma cells $(17,18)$. However, the expression of miR-214 has never been explored in patients with PCa that exhibit bone metastasis. To the best of our knowledge, the present study is the first to compare the expression levels of serum miR-214 in patients with PCa with bone metastasis, patients with $\mathrm{PCa}$ without bone metastasis and healthy controls, thereby assessing the potential of serum miR-214 expression as a diagnostic biomarker in patients with PCa with bone metastasis.

\section{Materials and methods}

Patients and blood samples. Serum samples of male patients with $\mathrm{PCa}$ with bone metastasis, non-bone metastasis, BPH group and healthy individuals were obtained from the Department of Urology, Changsha Central Hospital (Changsha, China) between June 2014 and January 2016. The application of patient-derived materials was approved by the Research Ethics Committee of Changsha Central Hospital (Changsha, China) and written informed consent was obtained from all patients. No patients received androgen-deprivation therapy or radiotherapy. Clinical characteristics of patients are listed in Table I. The differentiation of tumor cells was evaluated according to the Gleason score (GS) (19). Considering histological differentiation, the patients with PCa were then divided into 3 subgroups according to the GS: Well differentiated $(\mathrm{GS} \leq 6)$, moderately differentiated subgroup $(\mathrm{GS}=7)$, and poorly differentiated (GS $\geq 8$ ).

Cell lines and samples. The human PCa cell line PC3 was purchased from the Cell Bank of the Chinese Academy of Sciences (Shanghai, China). Cells were cultured in Dulbecco's modified Eagle's medium (Thermo Fisher Scientific, Inc., Waltham, MA, USA) supplemented with $10 \%$ fetal bovine serum (Hyclone; GE Healthcare Life Sciences, Logan, UT, USA), streptomycin $(100 \mu \mathrm{g} / \mathrm{ml})$ and penicillin $(100 \mathrm{U} / \mathrm{ml}$; Thermo Fisher Scientific, Inc.) in $25-\mathrm{cm}^{2}$ culture flasks at $37^{\circ} \mathrm{C}$ in a humidified atmosphere containing $5 \% \mathrm{CO}_{2}$.

Detection method. Serum BSP (cat. no. LP-001185, Shanghai Lanpai Bio. Tech. Co., Shanghai, China) and ICTP (cat. no. LP-001623, Shanghai Lanpai Bio. Tech. Co.,) levels were detected using ELISA. ALP activity was tested using the velocity method (20) (Nanjing Jiancheng Biological Technology Co., Ltd., Nanjing China) and the PSA level was determined using electrochemical immunoluminescence with a plate reader (Nanjing Jiancheng Biological Technology Co., Ltd.). Samples were read at a $450 \mathrm{~nm}$ wavelength using a microplate reader (Model 3550; Thermo Fisher Scientific, Inc.).

Cell transfection. PC3 cells were seeded in a 12- or 24-well plate for $48 \mathrm{~h}$. The following day, PC3 cells were transfected with the mature human miR-214 (5'-UGCCUGUCUACA CUUGCUGUGC-3'), anti-miR-214 (antisense inhibitor, 5'-CGACAGCAAGUGUAGACAGGCA-3'), small interfering RNA (siRNA) targeting phosphatase and tensin homolog (PTEN) (si-PTEN-1, 5'-AAGCTGGAA AGGGACGAA CT-3'; si-PTEN-2: 5'-GCTCAAGAGCAGCTACTACAT-3'; si-PTEN-3: 5'-UGCTCCGAACGTGTCACGT-3') or with a specific negative control siRNA (NC, 5'-CAGUACUUU GUGUAGUACAA-3') (all purchased from GeneChem, Inc., Daejeon, Korea) at a concentration of $50 \mathrm{nM}$ using Lipofectamine ${ }^{\circledR} 2000$ (Invitrogen; Thermo Fisher Scientific, Inc.), according to the manufacturer's protocol. For subsequent experiments, cells were used $48 \mathrm{~h}$ after transfection.

Western blot analysis. Total proteins were isolated from cells using a total protein extraction kit (cat no. KPG2100, Nanjing KeyGen Biotech Co., Ltd., Nanjing, China). A BCA protein assay kit (Pierce; Thermo Fisher Scientific, Inc.) was used to determine the protein concentration. Subsequently, supernatants were extracted from the lysates following centrifugation at $11,000 \mathrm{x} \mathrm{g}$ at $4^{\circ} \mathrm{C}$ for $15 \mathrm{~min}$. Equal amounts of protein (30 $\mu \mathrm{g} /$ lane) were separated using 10\% SDS-PAGE at $300 \mathrm{~mA}$ for $2 \mathrm{~h}$ and transferred onto a polyvinylidene fluoride membrane. Membranes were then blocked with $5 \%$ fat-free milk in Tris-buffered saline with $0.1 \%$ Tween-20 (TBST) buffer at room temperature for $2 \mathrm{~h}$. The membranes were then incubated with the following primary antibodies: Anti-PTEN (cat no. 9188; 1:1,000 dilution; Cell Signaling Technology, Inc., Danvers, MA, USA) and anti-GAPDH (cat no. 5174; 1:1,000 dilution; Cell Signaling Technology, Inc.) at $4^{\circ} \mathrm{C}$ overnight. Following three washes with TBST $(5 \mathrm{~min})$ at room temperature, the membranes were incubated with horseradish-peroxidase (HRP)-conjugated goat anti-rabbit secondary antibody (1:5,000; cat. no. ZB-2306, Zhongshan Gold Bridge Biological Technology Co., Beijing, China) for $2 \mathrm{~h}$ at room temperature and then washed with TBST three times for $5 \mathrm{~min}$ at room temperature followed by detection with enhanced chemiluminescent substrate (EMD Millipore, Billerica, MA, USA). GAPDH was used as a control. The proteins were detected using enhanced chemiluminescence (Super ECL Plus; Nanjing KeyGen Biotech Co., Ltd.) and densitometric analysis of the bands was performed using UVP automatic dyed gel free gel imaging system (GelDocIT TS2; UVP, LLC, Phoenix, AZ, USA). ImageJ 1.8.0 (National Institutes of Health, Bethesda, MD, USA) was applied to quantify the relative protein levels. The integral optical density ratio of PTEN/GAPDH indicated the relative expression of PTEN protein.

RNA extraction. Total RNA or miRNA from the total volume of serum samples $(5 \mathrm{ml})$ from patients with $\mathrm{PCa}$ with bone metastasis, non-bone metastasis, BPH group and healthy individuals was extracted using RNAzol ${ }^{\circledR}$ reagent (Vigorous Biotechnology Co., Ltd., Beijing, China), according to the manufacturer's protocol. The concentration and the purity of the RNA samples were assayed by determining the optical density ratio at 260/280 $\mathrm{nm}$.

Reverse transcription-quantitative polymerase chain reaction $(R T-q P C R)$. Total RNA $(10 \mu \mathrm{g})$ from the serum samples $(5 \mathrm{ml})$ 
Table I. Patient characteristics.

\begin{tabular}{|c|c|c|c|c|}
\hline Variable & Bone metastasis group & Non-bone group & Benign prostatic hyperplasia group & Healthy controls \\
\hline Total subjects, $\mathrm{n}$ & 75 & 65 & 45 & 56 \\
\hline Age, years (range) & $72(55-86)$ & $70(56-84)$ & $69(55-81)$ & $51(39-85)$ \\
\hline $\mathrm{BMI}, \mathrm{kg} / \mathrm{m}^{2}$ & $24.5 \pm 2.71$ & $24.3 \pm 3.04$ & $24.1 \pm 3.34$ & $24.5 \pm 2.18$ \\
\hline \multicolumn{5}{|l|}{ PSA, ng/ml } \\
\hline$<10, \mathrm{n}(\%)$ & $23(30.7)$ & $35(53.8)$ & $45(100.0)$ & $70(100.0)$ \\
\hline $10-20, \mathrm{n}(\%)$ & $25(33.3)$ & $10(15.4)$ & $0(0)$ & $0(0)$ \\
\hline$>20, \mathrm{n}(\%)$ & $27(36.0)$ & $12(18.5)$ & $0(0)$ & $0(0)$ \\
\hline \multicolumn{5}{|l|}{ Gleason score } \\
\hline$<7, \mathrm{n}(\%)$ & $21(28.0)$ & $17(26.2)$ & - & - \\
\hline $7, \mathrm{n}(\%)$ & $26(34.7)$ & $18(27.7)$ & - & - \\
\hline$>7, \mathrm{n}(\%)$ & $28(41.3)$ & $18(27.7)$ & - & - \\
\hline \multicolumn{5}{|l|}{ Tumor stage } \\
\hline $\mathrm{pT} 1 / 2, \mathrm{n}(\%)$ & $44(58.7)$ & $21(32.3)$ & - & - \\
\hline $\mathrm{pT} 3 / 4, \mathrm{n}(\%)$ & $31(41.3)$ & $22(33.8)$ & - & - \\
\hline \multicolumn{5}{|l|}{ Node stage } \\
\hline No, n (\%) & $41(54.7)$ & $25(38.5)$ & - & - \\
\hline $\mathrm{N} 1, \mathrm{n}(\%)$ & $34(45.3)$ & $22(33.8)$ & - & - \\
\hline \multicolumn{5}{|l|}{ Metastasis stage } \\
\hline $\mathrm{M} 0, \mathrm{n}(\%)$ & $40(53.3)$ & $46(70.8)$ & - & - \\
\hline $\mathrm{M} 1, \mathrm{n}(\%)$ & $35(46.7)$ & $29(44.6)$ & - & - \\
\hline
\end{tabular}

A Gleason score $<7$ is considered low grade; A Gleason score of 7 is considered intermediate grade and a Gleason score $>7$ is considered high grade. BMI, body mass index; p, pathological; T, tumor; N, node; M, metastasis.

was isolated using RNAVzol LS (Vigorous Biotechnology Co., Ltd., Beijing, China) according to the manufacturer's protocol. The concentration and purity of the RNA samples were determined by the $\mathrm{OD}_{260} / \mathrm{OD}_{280}$ ratio using a microplate reader (Model 3550; Thermo Fisher Scientific, Inc.). A total of $1 \mu \mathrm{g}$ RNA was reverse-transcribed using a TaqMan MicroRNA Reverse Transcription kit (Applied Biosystems; Thermo Fisher Scientific, Inc.) using specific primers for miR-214 and U6 (Sangon BioTech Co., Ltd., Shanghai, China). U6 is a small nuclear RNA employed as the endogenous control (21). The sequences of the nucleotide primers used for reverse transcription were as follows: miR-214, 5'-CGTATCCAGTGCAGG GTCCGAGGTATTCGCACTGGATACGACGCACAG-3'; U6, 5'-TCGTATCCAGTGCAGGGTCCGAGGTATTCGCACTGG ATACGACAAATATG-3'. The sequences for the primers used for qPCR were as follows: miR-214, forward, 5'-GCUGCCTGT CTACACTTG-3'; T6, forward, 5'-GCGTCGTGAAGCGTT C-3'; universal reverse primer, 5'-GTGCAGGGTCCGAGGT-3'.

The PCR amplifications were performed in a $10 \mu 1$ reaction system containing $5 \mu \mathrm{l} \mathrm{SYBR}$ Green Supermix, $0.4 \mu \mathrm{l}$ forward primer, $0.4 \mu \mathrm{l}$ reverse primer, $2.2 \mu \mathrm{l}$ double-distilled water and $2 \mu 1$ template cDNA using SYBR Green Supermix (Bio-Rad Laboratories, Inc., Hercules, CA, USA) in a Bio-Rad iCycler iQ Real-Time PCR Detection System. The thermal cycling conditions included an initial denaturation step at $95^{\circ} \mathrm{C}$ for $10 \mathrm{~min}$, followed by 40 cycles of $95^{\circ} \mathrm{C}$ for $15 \mathrm{sec}$ and final extension at $60^{\circ} \mathrm{C}$ for $1 \mathrm{~min}$. Relative levels of miR-214 were determined using the $2^{-\Delta \Delta \mathrm{Cq}}$ method (22).
Invasion assays. Cell invasion assays were performed using Boyden chambers ( $8-\mu \mathrm{m}$ pore filter; Corning Incorporated, Corning, NY, USA). For the cell invasion assay, 2.0x10 5 cells were cultured in a chamber (BD Biosciences, Franklin Lakes, NJ, USA) pre-coated with $0.2 \%$ Matrigel (BD Biosciences) at $37^{\circ} \mathrm{C}$. In the lower chamber, $10 \%$ fetal bovine serum was added to the culture medium as a chemoattractant. At $24 \mathrm{~h}$, the cells in the upper compartment were removed using cotton swabs and cells that had invaded through the membrane were stained with a dye solution containing $20 \%$ methanol for $30 \mathrm{~min}$ at $37^{\circ} \mathrm{C}$ and $0.1 \%$ crystal violet for $1 \mathrm{~h}$ at $37^{\circ} \mathrm{C}$. Images were captured under a light microscope (Olympus Corporation, Tokyo, Japan) and 10 individual fields were counted per insert. Three independent experiments were performed.

Statistical analysis. Data were analyzed using SPSS software (version 13.0; SPSS, Inc., Chicago, IL, USA). The relevant data are expressed as the mean \pm standard deviation. A two-tailed unpaired Student's t-test was used to examine differences between two groups. One-way analysis of variance followed by Tukey's post hoc test was used to examine differences among multiple groups. Receiver operating characteristic curve (ROC) curves were created and the areas under the curve (AUCs) were determined to assess the specificity and sensitivity of circulating miR-214 as a diagnostic biomarker. $\mathrm{P}<0.05$ was considered to indicate a statistically significant difference. 

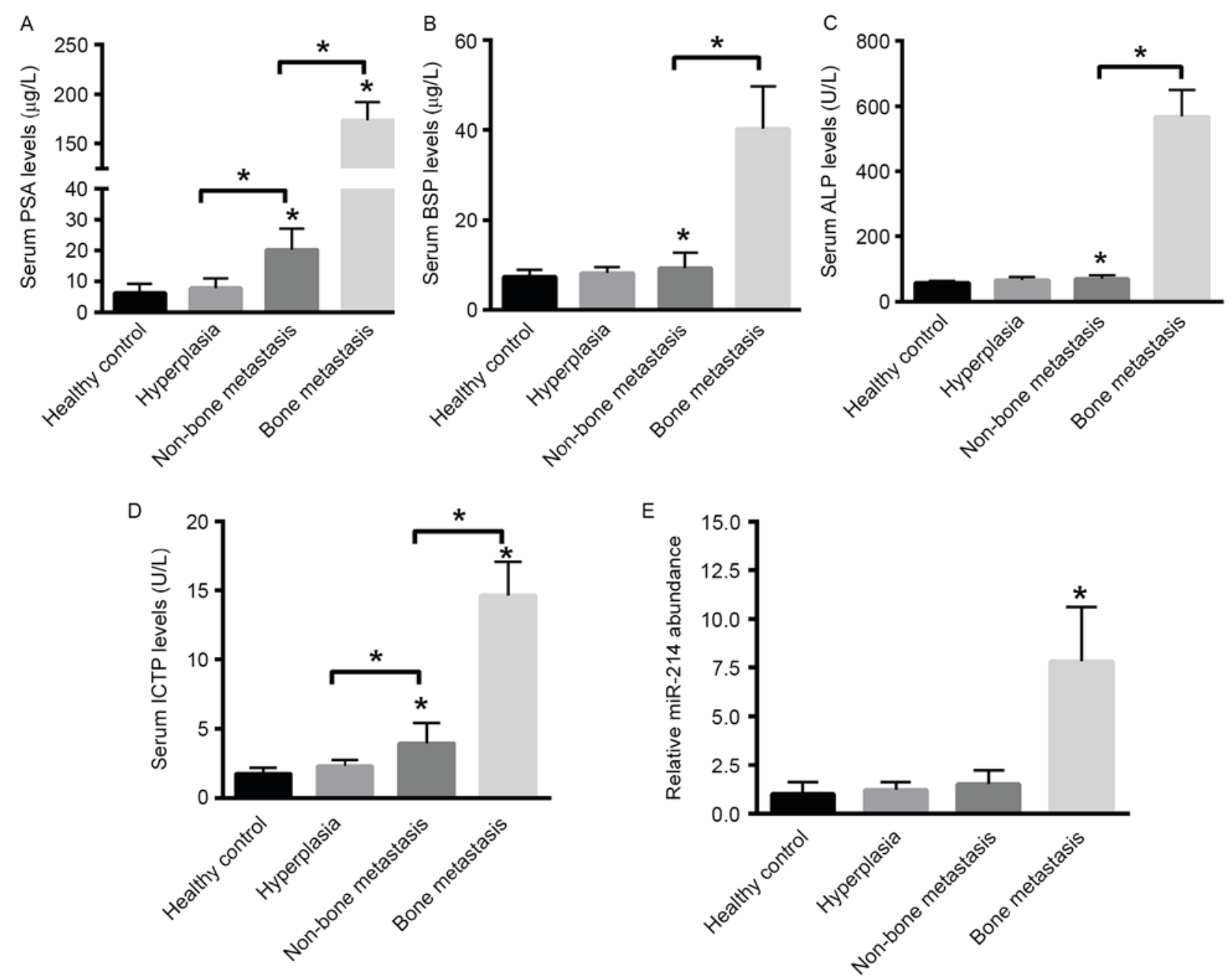

Figure 1. Enzyme-linked immunosorbent assay of PSA, BSP, ALP and ICTP levels and PCR analysis of serum miR-241 levels in patients with PCa with bone metastasis compared with the patients with PCa without bone metastasis, hyperplasia and healthy control groups. Expression levels of serum (A) PSA, (B) BSP, (C) ALP and (D) ICTP in the bone metastasis, non-bone metastasis, hyperplasia and healthy control groups. "P<0.05. (E) PCR analysis of relative expression levels of serum miR-214 in the bone metastasis, non-bone metastasis, hyperplasia and healthy control groups. * $\mathrm{P}<0.05$ vs. remaining groups. PSA, prostate-specific antigen; BSP, bone sialoprotein; ALP, alkaline phosphatase; ICTP, collagen type I pyridine crosslinking peptide; miR, microRNA; PCa, prostate cancer; PCR, polymerase chain reaction.

\section{Results}

Patient characteristics. As presented in Table I, the age distribution was similar among the healthy controls, patients with $\mathrm{BPH}$ and patients with $\mathrm{PCa}(\mathrm{P}>0.05)$. The expression levels of serum PSA were significantly upregulated in patients with PCa compared with in healthy controls $(\mathrm{P}<0.001)$.

Expression levels of miR-214, PSA, ALP, BSP and ICTP are significantly upregulated in the serum of patients with $P C a$ with bone metastasis. First, the expression levels of PSA, ALP, BSP and ICTP were evaluated in the serum of patients with PCa with or without bone metastasis, BPH group and healthy control group using ELISA. The results suggested that the serum levels of PSA, BSP, ALP and ICTP were increased in the bone metastasis group compared with in the non-bone metastasis, BPH and control groups $(\mathrm{P}<0.05$; Fig. 1A-D). Expression levels of miR-214 among the various groups were also evaluated using RT-qPCR. The results indicated that the expression level of miR-214 was significantly increased in the serum of patients with PCa with bone metastasis compared with that of non-bone metastasis, $\mathrm{BPH}$ and healthy control groups $(\mathrm{P}<0.001$; Fig. 1E). The results indicated that the relative expression of miR-214 among the various groups was as follows: $7.8 \pm 2.8$ for the bone metastasis group; $1.5 \pm 0.7$ for the non-bone metastasis group; $1.2 \pm 0.4$ for the $\mathrm{BPH}$ group and $1 \pm 0.6$ for the healthy control group. These data suggested that increased expression levels of miR-214 associated with significant bone metastasis in patients with PCa.

Increased expression levels of $m i R-214$ are associated with tumor malignancy in patients with PCa. The expression levels of PSA, BSP, ALP and ICTP were determined in well-differentiated (GS $<7)$, moderately differentiated $(\mathrm{GS}=7)$ and poorly differentiated tumors (GS $>7$ ) in patients with PCa. The results suggested that the expression of PSA, BSP, ALP and ICTP was significantly increased in the moderately differentiated and poorly differentiated tumors compared with well-differentiated tumors (Fig. 2A-D). As presented in Fig. 2E, increased expression levels of miR-214 were identified in the bone metastasis group exhibiting increased aggressive tumors in comparison with patients 
A

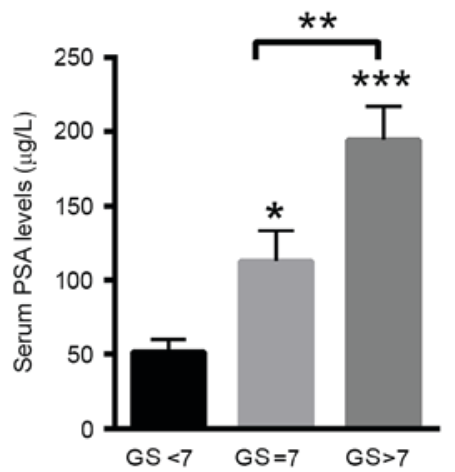

B

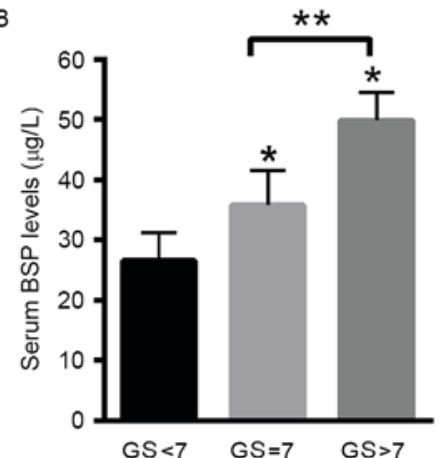

C

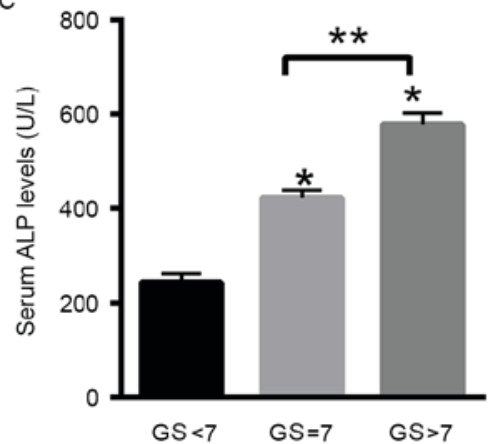

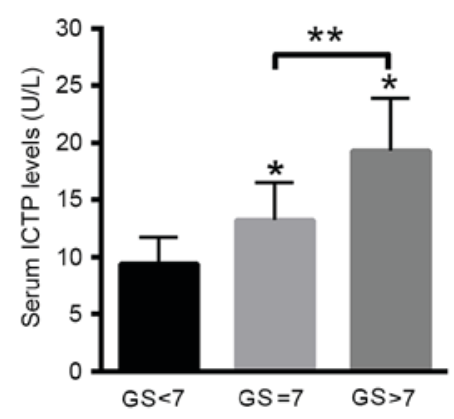

E
GS

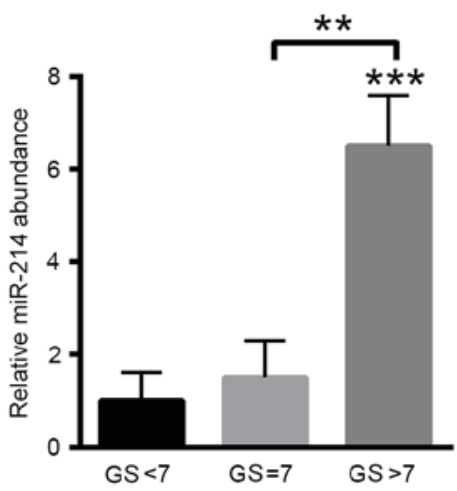

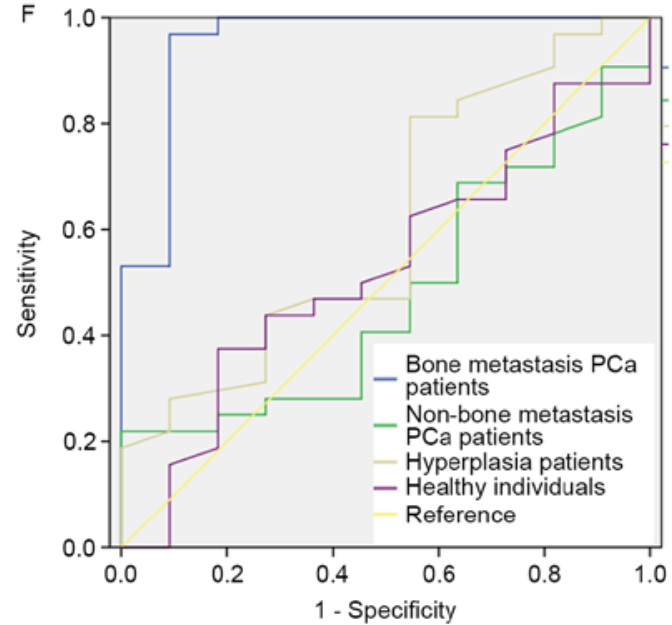

Figure 2. Increased expression levels of miR-214 are associated with clinicopathological parameters of patients with PCa. The expression levels of (A) PSA, (B) BSP, (C) ALP and (D) ICTP were analyzed in well-differentiated tumors (GS <7), moderately differentiated (GS=7) and poorly differentiated tumors (GS >7) of patients with PCa. (E) Relative expression levels of miR-214 in the serum of PCa patients with GS $<7, \mathrm{GS}=7$ and GS $>7$ of patients with PCa. ${ }^{* * *} \mathrm{P}<0.001$ vs. GS $<7 ;{ }^{* *} \mathrm{P}<0.01$ vs. GS=7; $\mathrm{P}<0.05$ vs. GS $<7$. (F) ROC analysis of miR-214 in patients with PCa with bone metastasis compared with the patients with PCa without bone metastasis, in hyperplasia and healthy control groups. Data are presented as the mean \pm standard deviation. PSA, prostate-specific antigen; BSP, bone sialoprotein; ALP, alkaline phosphatase; ICTP, collagen type I pyridine crosslinking peptide; miR, microRNA; PCa, prostate cancer; GS, Gleason score.

with less aggressive tumors. For patients with a GS $<7$, the relative expression level of miR-214 was $1.0 \pm 0.6$. For patients with a GS of 7, the relative expression level of miR-214 was increased to $1.5 \pm 0.8$, whereas the relative expression of miR-214 was increased to $2.5 \pm 1.1$ in patients with a GS $>7$ (Fig. 2E). ROC analysis indicated that serum levels of miR-214 were able to distinguish the bone metastasis group [AUC, 0.955; 95\% confidence interval (CI), 0.872-1.000; $\mathrm{P}<0.001]$ from the non-bone metastasis group (AUC, 0.479; 95\% CI, 0.291-0.667), BPH (AUC, 0.611; 95\% CI, 0.415-0.807) and healthy control group (AUC, $0.526 ; 95 \% \mathrm{CI}$, $0.332-0.719$ ). Additionally, miR-214 was able to distinguish patients with PCa from healthy individuals (AUC, 0.915; 95\% CI, 0.846-0.984; Fig. 2F).

PTEN is a target gene of miR-214 in PC3 cells. Next, the potential molecular mechanism by which miR-214 modulates bone metastasis in PCa was investigated. It has been demonstrated that PTEN is a target gene in various tumors, including gastric cancer and lymphocytic leukemia $(23,24)$. Then, whether miR-214 was able to target PTEN in PCa cells was investigated. As presented in Fig. 3A, overexpression of miR-214 significantly suppressed the protein level of PTEN in PC3 cells. In contrast, inhibition of miR-214 markedly increased the expression of PTEN in PC3 cells (Fig. 3B).
Suppression of PTEN increases the invasive ability of $P C a$ cells. The function of PTEN on the invasive ability of PCa cells was evaluated using PC3 cells. First, three specific siRNAs targeting PTEN were selected, but only two of them significantly suppressed the mRNA levels of PTEN (Fig. 4A). Thus, si-PTEN-1 and si-PTEN-2 were selected for subsequent experiments. Furthermore, the silencing of PTEN significantly increased the invasive ability of PC3 cells (Fig. 4B). Downregulation of miR-214 using miR-214i significantly suppressed the invasive ability of PCa cells. However, such effects were eliminated in response to si-PTEN transfection, thus suggesting that miR-214 functions as an oncogenic miRNA partially by targeting PTEN (Fig. 4C).

\section{Discussion}

Bone metastasis is a common complication in patients with $\mathrm{PCa}$ which markedly induces bone pain and pathological fracture $(25,26)$. However, the diagnostic tools for PCa are relatively limited. Previous studies have suggested that miRNAs may be potential diagnostic markers considering their key function in different conditions, including in cardiovascular disease, hepatocarcinoma and breast cancer (27-29). miRNAs are identified to be dysregulated through their oncogenic and tumor suppressive functions in the tumor $(27,29)$. Thus, it is necessary to investigate 

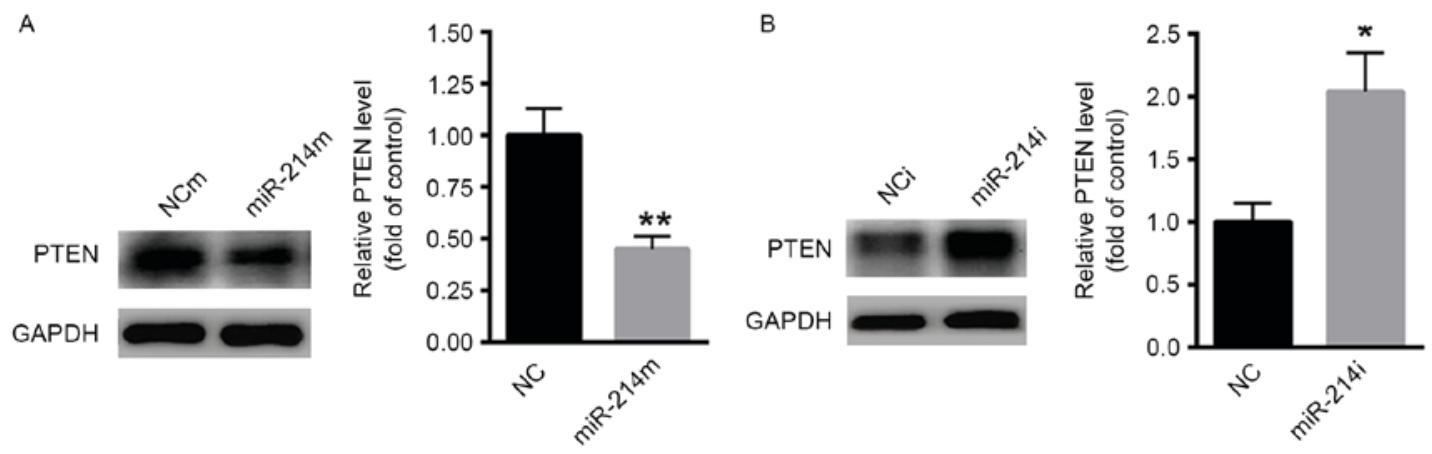

Figure 3. PTEN is a target gene of miR-214. (A) Overexpression of miR-214 significantly suppressed the protein level of PTEN. (B) Inhibition of miR-214 markedly increased the expression of PTEN in PC3 cells. ${ }^{*} \mathrm{P}<0.05,{ }^{* * *} \mathrm{P}<0.01$, vs. control. miR, microRNA; NC, negative control; PTEN, phosphatase and tensin homolog; I, inhibitor; m, mimic.

A

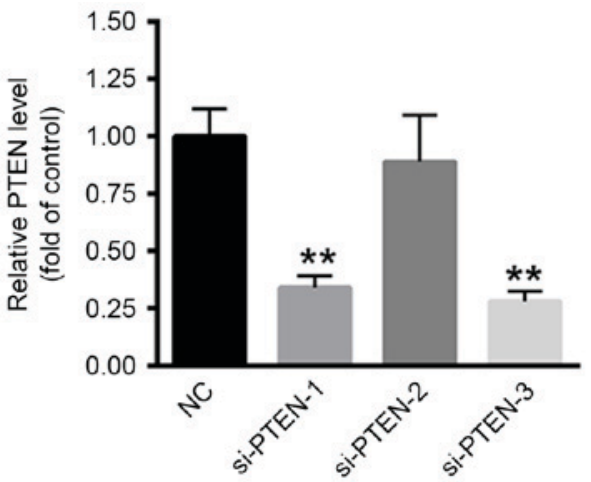

B

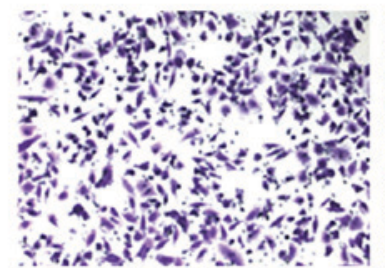

NC

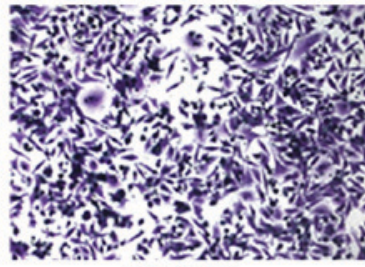

SI-PTEN-1

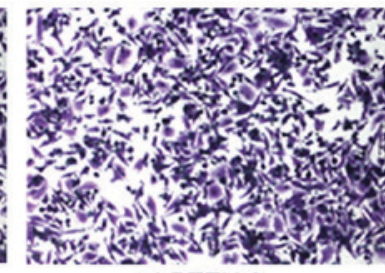

C

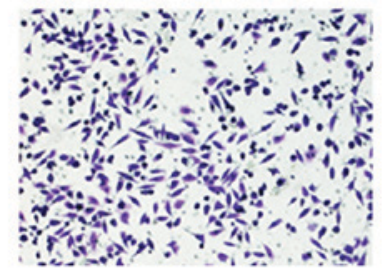

NC

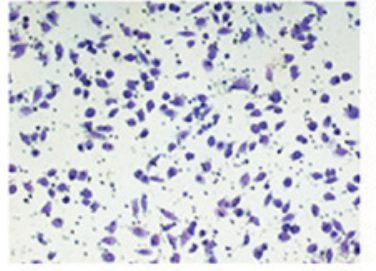

miR-214i

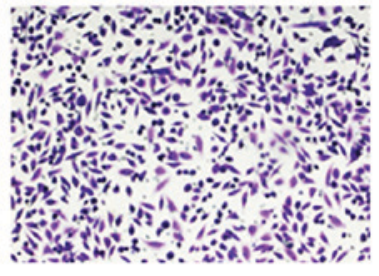

miR-214i+si-PTEN-1

Figure 4. Downregulation of PTEN increases the invasion of prostate cancer cells. (A) Western blot analysis of three sets of siRNAs targeting PTEN (si-PTEN-1, si-PTEN-2 and si-PTEN-3). (B) Silencing of PTEN significantly increased the invasive ability of PC3 cells. (C) Transfection of miR-214 inhibitor significantly decreased the invasive ability of PC 3 cells. ${ }^{* *} \mathrm{P}<0.01$, vs. control. miR, microRNA; siRNA/si, small interfering RNA; NC, negative control; PTEN, phosphatase and tensin homolog; I, inhibitor.

the function of miRNAs in the development of bone metastasis in patients with $\mathrm{PCa}$, which may lead to novel diagnostic tools for screening bone metastasis in patients with $\mathrm{PCa}$.

Previous studies have demonstrated that abnormal expression of miR-214 may enhance or suppress tumor progression in various types of tumors, including breast cancer, testicular germ cell tumor, hepatocellular carcinoma and gastric cancer (24,30-32). Furthermore, miR-214 increased the proliferative capacity of T cells by targeting PTEN (33). In the present study, the expression of miR-214 in the serum of patients with PCa with bone metastasis was investigated. The results indicated that miR-214 was significantly upregulated in the serum of patients with PCa with bone metastasis compared with the non-bone metastasis, BPH and healthy control groups. However, a previous study has indicated that the expression level of miR-214 was decreased in the urine samples of patients with $\mathrm{PCa}$ (34). This inconsistency may be because miR-214 is specifically upregulated in the serum of patients with bone metastasis.

Expression levels of additional serum bone markers were determined. For instance, GS $\geq 8$, PSA $\geq 20 \mathrm{mg} / 1$ and an increased ALP level are important indicators for bone metastases in patients with PCa $(35,36)$. In the present study, it was 
demonstrated that the serum levels of PSA, BSP, ALP and ICTP were increased in the bone metastasis group compared with the non-bone metastasis group, BPH and the control group. However, these serum bone markers were increased in all patients with PCa. Additionally, it was demonstrated that miR-214 may be increased only in patients with PCa with bone metastasis. However, increased expression of miR-214 was also associated with the malignant phenotype of $\mathrm{PCa}$, suggesting that its increased specificity and sensitivity may hold promise for its potential as a biomarker in patients with PCa with bone metastasis. Additionally, ROC analysis demonstrated that miR-214 may significantly distinguish the bone metastasis group from the other groups, indicating the potential for miR-214 as a biomarker in such patients.

miRNAs exert their function mainly through binding the $3^{\prime}$ untranslated region of target genes. Therefore, the potential target genes of miR-214 were investigated. Previous studies have demonstrated that PTEN is a target gene in various tumors, including gastric cancer and lymphocytic leukemia $(23,24)$. In the present study, it was demonstrated that overexpression of miR-214 significantly suppressed the protein levels of PTEN. Additionally, silencing of PTEN increased the invasive ability of PC 3 cells, even when miR-214 was inhibited. These results suggested that miR-214 increased the invasive ability of $\mathrm{PCa}$ cells through targeting PTEN.

In the present study, the statistical power may be restricted due to the limited number of experimental samples. Thus, it is necessary to incorporate several large prospective studies to determine the function of miR-214 in patients with $\mathrm{PCa}$ with bone metastasis. Despite this limitation, the results of the present study have identified a novel potential non-invasive biomarker for the diagnosis of PCa with bone metastasis.

\section{Acknowledgements}

Not applicable.

\section{Funding}

The present study was supported by a grant from the Hunan Province Supporting fund of Changsha Central Hospital (grant no. HNCS-20170623).

\section{Availability of data and materials}

The datasets used and/or analyzed during the current study are available from the corresponding author on reasonable request.

\section{Authors' contributions}

YF performed the experiments and analyzed the data. JQ, ZJ, SX and ZZ performed part of the RT-qPCR experiments. RH designed the experiments, analyzed the data and gave final approval of the version to be published. All authors read and approved the final manuscript.

\section{Ethics approval and consent to participate}

The present study was approved by the Research Ethics Committee of Changsha Central Hospital (Changsha, China) and all the patients provided written informed consent for this study.

\section{Consent for publication}

Not applicable.

\section{Competing interests}

The authors declare that they have no competing interests.

\section{References}

1. Siegel RL, Miller KD and Jemal A: Cancer statistics, 2015. CA Cancer J Clin 65: 5-29, 2015.

2. Siegel RL, Fedewa SA, Miller KD, Goding-Sauer A, Pinheiro PS, Martinez-Tyson D and Jemal A: Cancer statistics for Hispanics/Latinos, 2015. CA Cancer J Clin 65: 457-480, 2015

3. Luna A, Vilanova JC and Alcalá Mata L: Total body MRI in early detection of bone metastasis and its indication in comparison to bone scan and other imaging techniques. Arch Esp Urol 68: 371-390, 2015 (In Spanish).

4. Ramankulov A, Lein M, Kristiansen G, Loening SA and Jung K: Plasma osteopontin in comparison with bone markers as indicator of bone metastasis and survival outcome in patients with prostate cancer. Prostate 67: 330-340, 2007.

5. Draisma G, Etzioni R, Tsodikov A, Mariotto A, Wever E, Gulati R, Feuer E and de Koning H: Lead time and overdiagnosis in prostate-specific antigen screening: Importance of methods and context. J Natl Cancer Inst 101: 374-383, 2009.

6. Kim EH and Andriole GL: Prostate-specific antigen-based screening: Controversy and guidelines. BMC Med 13: 61, 2015.

7. Kitagawa $\mathrm{Y}$ and Namiki M: Prostate-specific antigen-based population screening for prostate cancer: Current status in Japan and future perspective in Asia. Asian J Androl 17: 475-480, 2015.

8. Kitajima K, Murphy RC, Nathan MA, Froemming AT, Hagen CE, Takahashi N and Kawashima A: Detection of recurrent prostate cancer after radical prostatectomy: Comparison of 11C-choline PET/CT with pelvic multiparametric MR imaging with endorectal coil. J Nucl Med 55: 223-232, 2014.

9. ChunJiao S, Huan C, ChaoYang X and GuoMei R: Uncovering the roles of miRNAs and their relationship with androgen receptor in prostate cancer. IUBMB Life 66: 379-386, 2014.

10. Pekarik V, Gumulec J, Masarik M, Kizek R and Adam V: Prostate cancer, miRNAs, metallothioneins and resistance to cytostatic drugs. Curr Med Chem 20: 534-544, 2013.

11. Stuopelytė K, Daniūnaitė K, Jankevičius F and Jarmalaite S: Detection of miRNAs in urine of prostate cancer patients. Medicina (Kaunas) 52: 116-124, 2016.

12. Sun T, McKay R, Lee GS and Kantoff P: The role of miRNAs in prostate cancer. Eur Urol 68: 589-590, 2015.

13. Chang YS, Chen WY, Yin JJ, Sheppard-Tillman H, Huang J and Liu YN: EGF receptor promotes prostate cancer bone metastasis by downregulating miR-1 and activating TWIST1. Cancer Res 75: 3077-3086, 2015.

14. Chen WY, Liu SY, Chang YS, Yin JJ, Yeh HL, Mouhieddine TH, Hadadeh O, Abou-Kheir W and Liu YN: MicroRNA-34a regulates WNT/TCF7 signaling and inhibits bone metastasis in Ras-activated prostate cancer. Oncotarget 6: 441-457, 2015.

15. Xin R, Bai F, Feng Y, Jiu M, Liu X, Bai F, Nie Y and Fan D: MicroRNA-214 promotes peritoneal metastasis through regulating PTEN negatively in gastric cancer. Clin Res Hepatol Gastroenterol 40: 748-754, 2016.

16. Kuninty PR, Bojmar L, Tjomsland V, Larsson M, Storm G, Östman A, Sandström P and Prakash J: MicroRNA-199a and -214 as potential therapeutic targets in pancreatic stellate cells in pancreatic tumor. Oncotarget 7: 16396-16408, 2016.

17. Das F, Dey N, Bera A, Kasinath BS, Ghosh-Choudhury N and Choudhury GG: MicroRNA-214 reduces insulin-like growth factor-1 (IGF-1) receptor expression and downstream mTORC1 signaling in renal carcinoma cells. J Biol Chem 291: 14662-14676, 2016.

18. Tang SL, Gao YL, and Chen XB: MicroRNA-214 targets PCBP2 to suppress the proliferation and growth of glioma cells. Int J Clin Exp Pathol 8: 12571-12576, 2015. 
19. Siemińska L, Borowski A, Marek B, Nowak M, Kajdaniuk D, Warakomski J and Kos-Kudła B: Serum concentrations of adipokines in men with prostate cancer and benign prostate hyperplasia. Endokrynol Pol: Feb 21, 2018 (Epub ahead of print).

20. Jansson UH, Kristiansson B, Magnusson P, Larsson L, Albertsson-Wikland $\mathrm{K}$ and Bjarnason R: The decrease of IGF-I, IGF-binding protein-3 and bone alkaline phosphatase isoforms during gluten challenge correlates with small intestinal inflammation in children with coeliac disease. Eur J Endocrinol 144: 417-423, 2001

21. Ofori JK, Salunkhe VA, Bagge A, Vishnu N, Nagao M, Mulder H, Wollheim CB, Eliasson L and Esguerra JL: Elevated $\mathrm{miR}-130 \mathrm{a} / \mathrm{miR} 130 \mathrm{~b} / \mathrm{miR}-152$ expression reduces intracellular ATP levels in the pancreatic beta cell. Sci Rep 7: 44986, 2017.

22. Livak KJ and Schmittgen TD: Analysis of relative gene expression data using real-time quantitative PCR and the 2(-Delta Delta C(T)) method. Methods 25: 402-408, 2001

23. Zou ZJ, Fan L, Wang L, Xu J, Zhang R, Tian T, Li JY and Xu W: miR-26a and miR-214 down-regulate expression of the PTEN gene in chronic lymphocytic leukemia, but not PTEN mutation or promoter methylation. Oncotarget 6: 1276-1285, 2015.

24. Yang TS, Yang XH, Wang XD, Wang YL, Zhou B and Song ZS MiR-214 regulate gastric cancer cell proliferation, migration and invasion by targeting PTEN. Cancer Cell Int 13: 68, 2013.

25. Fujii T, Shimada K, Tatsumi Y, Fujimoto K and Konishi N: Syndecan-1 responsive microRNA-126 and 149 regulate cell proliferation in prostate cancer. Biochem Biophys Res Commun 456: 183-189, 2015.

26. Fu H, He HC, Han ZD, Wan YP, Luo HW, Huang YQ, Cai C, Liang YX, Dai QS, Jiang FN and Zhong WD: MicroRNA-224 and its target CAMKK2 synergistically influence tumor progression and patient prognosis in prostate cancer. Tumour Biol 36: 1983-1991, 2015

27. Kloosterman WP and Plasterk RH: The diverse functions of microRNAs in animal development and disease. Dev Cell 11: 441-450, 2006.
28. Ha TY: MicroRNAs in human diseases: From cancer to cardiovascular disease. Immune Netw 11: 135-154, 2011.

29. Baranwal S and Alahari SK: miRNA control of tumor cell invasion and metastasis. Int J Cancer 126: 1283-1290, 2010.

30. Liu B, Tian Y, Li F, Zhao Z, Jiang X, Zhai C, Han X and Zhang L: Tumor-suppressing roles of miR-214 and miR-218 in breast cancer. Oncol Rep 35: 3178-3184, 2016.

31. Chen BF, Suen YK, Gu S,Li L and Chan WY: A miR-199a/miR-214 self-regulatory network via PSMD10, TP53 and DNMT1 in testicular germ cell tumor. Sci Rep 4: 6413, 2014.

32. Yin Y, Cai X, Chen X, Liang H, Zhang Y, Li J, Wang Z, Chen X, Zhang W, Yokoyama S, et al: Tumor-secreted miR-214 induces regulatory T cells: A major link between immune evasion and tumor growth. Cell Res 24: 1164-1180, 2014.

33. Jindra PT, Bagley J, Godwin JG and Iacomini J: Costimulation-dependent expression of microRNA-214 increases the ability of $\mathrm{T}$ cells to proliferate by targeting Pten. J Immunol 185: 990-997, 2010.

34. Srivastava A, Goldberger H, Dimtchev A, Ramalinga M, Chijioke J, Marian C, Oermann EK, Uhm S, Kim JS, Chen LN, et al: MicroRNA profiling in prostate cancer-the diagnostic potential of urinary miR-205 and miR-214. PLoS One 8: e76994, 2013

35. Kamiya N, Suzuki H, Yano M, Endo T, Takano M, Komaru A, Kawamura K, Sekita N, Imamoto T and Ichikawa T: Implications of serum bone turnover markers in prostate cancer patients with bone metastasis. Urology 75: 1446-1451, 2010.

36. Moslehi M, Cheki M, Salehi-Marzijarani M, Amuchastegui T and Gholamrezanezhad A: Predictors of bone metastasis in pre-treatment staging of asymptomatic treatment-naive patients with prostate cancer. Rev Esp Med Nucl Imagen Mol 32: 286-289, 2013 . 